\title{
EFEITO DA SOLARIZAÇÃO SOBRE PROPRIEDADES FíSICAS, QUÍMICAS E BIOLÓGICAS DE SOLOS ${ }^{(1)}$
}

\author{
R. GHINI(2), F.R.A. PATRICIO(3), M.D. SOUZA(4), C. SINIGAGLIA(3), B.C. BARROS(3), \\ M. E. B. M. LOPES ${ }^{(3)}$, J . TESSARIOLI NETO(5) \& H. CANTARELLA ${ }^{(6)}$
}

\begin{abstract}
RESUMO
A solarização é um método de desinfestação que consiste na cobertura do solo com um filme de polietileno transparente, durante o período de intensa radiação solar, e atua por meio do aumento da temperatura do solo. Quatro ensaios foram realizados no estado de São Paulo, nos municípios de Mogi das Cruzes, J arinu, Piracicaba e I tati ba, nos anos de 2000 e 2001, com o objetivo de avaliar os efeitos da solarização nas propriedades físicas, químicas e biológicas dos solos. A solarização reduzi u si gnificativamente a resistência à penetração dos solos nos ensaios de J arinu, Piracicaba e Itatiba. Em J arinu, oito meses após a retirada do plástico, as diferenças entre os tratamentos permaneceram. Por outro lado, em Mogi das Cruzes, onde o ensaio foi instalado em solo turfoso, a solarização causou aumento na resistência na camada de 2,5 a $5 \mathrm{~cm}$ de profundidade. Nos ensaios de Piracicaba e J arinu, foram feitas avaliações de macro, microporosidade, porosi dade total e densidade, não tendo os tratamentos diferido entre si, porém houve uma tendência de redução na densidade dos solos solarizados. A atividade microbiana, avaliada pela hidrólise de diacetato de fluoresceína, foi reduzida pela solarização. A supressividade a Fusarium oxysporum f. sp. phaseoli foi avaliada in vitro, pela colonização de amostras de solo usando um isolado marcado com resistência a benomyl, no ensaio de Mogi das Cruzes. A solarização reduziu a recuperação do patógeno, evidenciando um aumento da supressividade. Nos solos solarizados, houve aumento significativo dos teores de $\mathrm{N}-\mathrm{NH}_{4}{ }^{+}$em todos os experimentos, $\mathrm{Mn}$ em três, $\mathrm{N}-\mathrm{NO}_{3}{ }^{-}, \mathrm{Mg}^{2+}$ e saturação por bases em dois e $\mathrm{K}^{+}$em um experimento. Ocorreu redução dos teores de $\mathrm{Cu}$, Fe e H + Al em dois experimentos e Zn em um ensaio. Segundo os resultados, a solarização promoveu alterações nas propriedades físicas, químicas e biológicas dos solos, melhorando a estrutura, liberando nutrientes e aumentando a supressividade.
\end{abstract}

Termos de indexação: resistência à penetração, supressividade, controle físico.

(1) Parte do trabal ho apresentado no XXXIV Congresso Brasileiro de Fitopatologia, São Pedro, agosto 2001. Trabal ho financiado pela FAPESP. Recebido para publicação em janeiro de 2002 e aprovado em setembro de 2002.

(2) Pesquisador da Embrapa Meio Ambiente. Caixa Postal 69, CEP 13820-000 J aguariúna (SP). Bolsista do CNPq. E-mail: raquel@cnpma.embrapa.br

(3) Pesquisador do Instituto Biológico. Caixa Postal 70, CEP 13001-970 Campinas (SP). E-mail: flavia@biologico.br; cel so@biologico.br; bcbarros@giologico.br; mebmlopes@yahoo.com

(4) Pesquisador da Embrapa Meio Ambiente. Caixa Postal 69, CEP 13820-000 J aguariúna (SP).E-mail: dornelas@cnpma.embrapa.br

(5) Professor da Escola Superior de Agricultura "Luiz de Queiroz" - ESALQ/USP. Caixa Postal 9, CEP 13418-900 Piracicaba (SP). E-mail: jtessari@esalq.usp.br

(6) Pesquisador do Instituto Agronômico - IAC. Caixa Postal 28, CEP 13001-970 Campinas (SP). E-mail: hcantare@iac.br 


\title{
SUMMARY: SOLARIZATION EFFECTS ON PHYSICAL, CHEMICAL AND BIOLOGICAL PROPERTIES OF SOILS
}

\begin{abstract}
Solarization is a method for soil disinfestation, taking effect by increased soil temperatures under soil covers of transparent pol yethylenesheets during theperiod of intense solar irradiation. Four field experiments were set up in the state of São Paulo, Brazil (districts of Mogi das Cruzes, J arinú, Piracicaba and Itati ba), in the years 2000 and 2001, to eval uatetheeffects of soil solarizati on on physical, chemical and biol ogical soil properties. Solarization caused a significant reduction in penetration resistance of the eval uated soils in J arinu, Piracicaba, and I tatiba. In J arinu, measurements carried out eight months after the removal of the plastic sheet showed that the effect of solarization on penetration resistance was still similar to that observed in the first evaluation. However, in Mogi das Cruzes, in an organic soil, solarization increased the soil's penetration resistance in the 2.5-5.0 cm layer. Sol arization did not affect themacro-, micro- and total porosity of soil s in Piracicaba and J arinu, where these properties were measured, however it did reduce the apparent density. In an evaluation by fluorescein diacetatehydrolysis, themi crobial activity in the soil proved to decrease by sol arization. In Mogi das Cruzes, the suppressi veness to Fusarium oxysporum f. sp. phaseoli was eval uated in vitro by col onizing soil samples with an isol atemarked with benomyl resistance. Sol arization reduced therecovery of thepathogen, indicating an increasein suppressiveness. In solarized plots, therewas a significant increase in the concentration of $\mathrm{NH}_{4}{ }^{+}-\mathrm{N}$ in all experiments, of $\mathrm{Mn}$ in three, of $\mathrm{NO}_{3}{ }^{-} \mathrm{N}, \mathrm{Mg}^{2+}$, and base saturation in two, and $\mathrm{K}^{+}$in one experiment. On the other hand, the concentration of $\mathrm{Cu}$, $\mathrm{Fe}$, and $\mathrm{H}+\mathrm{Al}$ decreased in two places, and $\mathrm{Zn}$ in one. Results showed that solarization caused changes in physical, chemical and biological soil properties, improving the soil structure, releasing nutrients, and increasing disease suppressi veness.
\end{abstract}

Index terms: resistanceto penetration, suppressiveness, physical control.

\section{INTRODUÇÃO}

A solarização é um método de desinfestação do solo, que foi desenvolvido, em I srael, por Katan et al. (1976), e vem sendo utilizado em diversos países, induindo oBrasil (Katan \& DeVay, 1991; Souza, 1994; Ghini \& Bettiol, 1995). A técnica consiste na cobertura do solo úmido, com um filme plástico transparente, antes do plantio, preferencialmente durante o período de maior incidência de radiação solar.

A solarização objetiva controlar fitopatógenos veiculados pelo solo. A cobertura com o plástico promove o aquecimento, especial mente das camadas superficiais do solo, inibindo ou eliminando organismos. Assim, parte da população de patógenos é morta em decorrência da el evação da temperatura. Todavia, apesar de a exposição direta dos patógenos ao calor ser importante fator, não é o único mecanismo envolvido no método. Os processos microbianos induzidos pela solarização contribuem para o control e de doenças, já que o aquecimento atua também sobre organismos nãovisados (Katan, 1981).

Geralmente, os mi crorganismos saprófitas do solo, dentre eles inúmeros antagonistas, são mais tolerantes ao calor do que os fitopatógenos. A mudança na microbiota do solo em favor de antagonistas aumenta a eficiência do controle, especialmentenas camadas mais profundas do solo, já que o enfraquecimento dos propágulos dos patógenos pelas temperaturas subletais é seguido pelo ataque dos antagonistas (Lifshitz et al., 1983). Assim, uma vantagem do método é que essa mudança na população microbiana pode levar à supressividade a patógenos, tendendo a proteger o solo de uma reinfestação e garantindo um efeito mais duradouro do tratamento (Katan \& DeVay, 1991).

Outros efeitos, porém, podem ser observados após o tratamento. Diversas plantas invasoras também podem ser controladas pela solarização (Bettiol et al., 1994). Em muitas hortas comerciais, a solarização está sendo utilizada apenas no controle das plantas invasoras, visto que significa uma redução de mão-de-obra, como no caso da alface (Sinigaglia et al., 2001).

O maior crescimento de plantas éfreqüentemente observado nos sol os solarizados, assim como maior produtividade. Esse efeito, que pode ocorrer mesmo na ausência de patógenos, deve-se a diversos processos desenvol vidos durante a solarização, que envolvem mudanças nos componentes bióticos e abióticos do sol o (Katan \& DeVay, 1991; Ghini et al., 1992; Gruenzweig et al., 1993). O mai or crescimento é resultado do controle de pragas ou patógenos primários e, ou, secundários, alteraçãoda comunidade 
microbiana do solo em favor de antagonistas ou microrganismos promotores de crescimento, inativação térmica de plantas invasoras e liberação de nutrientes no solo, decorrentes da morte e decomposição de parte da microbiota.

Essas alterações, al ém de outras, como mudanças na composição gasosa do solo, liberação de substâncias voláteis, melhoria da estrutura e penetração profunda da umidade, constituem um processo integrado que altera o ambiente do solo, resultando em maior crescimento e produção de plantas (Katan, 1996). Apesar deserem esses efeitos freqüentemente observados, poucos trabalhos estudam e quantificam tais alterações físicas, químicas e biológicas causadas pela solarização.

O comportamento de cada ti po de sol o em relação à solarização depende dos materiais constituintes, pois, segundo Prevedello (1996), a quantidade de cal or que pode ser transferida por condução no solo depende da sua condutividade térmica e da quantidade de energia térmica que uma massa ou vol ume desoloarmazena antes que sua temperatura se eleve (calor específico gravimétrico ou volumétrico). O tempo requerido para determinado solo aumentar a temperatura depende de como o cal or étransmitido e do cal or específico de cada fase constituinte do solo (sólida, líquida e gasosa). Por exemplo, a fração mineral do solo apresenta um cal or específico gravimétrico aproximado de $712 \mathrm{~J} /(\mathrm{K} \mathrm{kg})$, a orgânica de $1.926 \mathrm{~J} /(\mathrm{K} \mathrm{kg})$, a água de $4.186 \mathrm{~J} /(\mathrm{K} \mathrm{kg})$ e o ar de $1.005 \mathrm{~J} /(\mathrm{K} \mathrm{kg})$. Portanto, para cada tipo de solo, tem-se um comportamento diferenciado na variação de temperatura.

Assim sendo, o presentetrabalho teve por objetivo avaliar os efeitos da solarização em características físicas, químicas e biológicas de sol os em diferentes agroecossistemas. Dentre os fatores físicos, a resistência à penetração no solo após a solarização foi estudada por não haver relatos na literatura sobre os efeitos do tratamento nessa característica. Além disso, também foram estudadas a porosidade e a densidade dos solos. A análise de fertilidadefoi feita para verificar os efeitos nas características químicas. Os fatores biológicos estudados foram a atividade microbiana e a indução de supressividade a Fusarium oxysporum f. sp. phaseoli, que constitui um patógeno veiculado pelo solo de ocorrência cosmopolita.

\section{MATERIAL E MÉTODOS}

Quatro experimentos com a solarização do solo for am instal ados no estado de São Paulo, nas regi ões de Mogi das Cruzes, J arinu, Piracicaba el tatiba, nos anos de 2000 e 2001 (Quadro 1).

\section{Mogi das Cruzes}

O experimento foi instalado em uma área comercial, produtora de alface, infestada com Sclerotinia minor e Rhizoctonia solani, situada numa várzea drenada, com solo turfoso. O experimento foi em blocos casualizados, com quatro repetições. Os tratamentos constituíram-se da combinação de dois fatores: solarização (solarizado ou não) e tratamento químico para $S$. minor e $R$. solani (duas aplicações de pencycuron na dose de $500 \mathrm{~g} \mathrm{ha}^{-1}$ de i.a.; duas aplicações de procimidone na dose de $600 \mathrm{~g}$ ha-1 de i.a.; esem tratamento químico), totalizando seis tratamentos.

Como o plantio dealfaceé realizado em canteiros, foram solarizadas parcelas com $4 \mathrm{~m}$ de largura, compostas por três canteiros de $1 \mathrm{~m}$ de largura e $20 \mathrm{~cm}$ entre os canteiros e $4 \mathrm{~m}$ de comprimento. $\mathrm{O}$ plástico, com espessura de $100 \mu \mathrm{m}$ ecom aditivo que absorve a radiação ultravioleta (UV) do sol, foi colocado sobre o solo em 21/12/1999 e retirado em

\section{Quadro 1. Caracterização granulométrica dos solos}

\begin{tabular}{|c|c|c|c|c|c|c|}
\hline \multirow{2}{*}{ Ensaio } & \multirow{2}{*}{ Profundidade } & \multicolumn{5}{|c|}{ Análise textural } \\
\hline & & Areia grossa & Areia fina & Silte & Argila & Textura \\
\hline & $\mathrm{cm}$ & 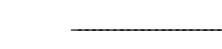 & -2 & 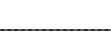 & - & \\
\hline Mogi das Cruzes & $\begin{array}{r}0-10 \\
10-20\end{array}$ & $\begin{array}{r}9,65 \\
19,45\end{array}$ & $\begin{array}{l}13,04 \\
25,41\end{array}$ & $\begin{array}{l}68,39 \\
39,30\end{array}$ & $\begin{array}{r}8,92 \\
15,85\end{array}$ & $\begin{array}{l}\text { Franco-siltosc } \\
\text { Franco }\end{array}$ \\
\hline J arinu & $\begin{array}{r}0-10 \\
10-20\end{array}$ & $\begin{array}{l}18,92 \\
20,13\end{array}$ & $\begin{array}{l}17,25 \\
17,52\end{array}$ & $\begin{array}{l}18,64 \\
17,45\end{array}$ & $\begin{array}{l}45,20 \\
44,89\end{array}$ & $\begin{array}{l}\text { Argila } \\
\text { Argila }\end{array}$ \\
\hline Piracicaba & $\begin{array}{r}0-10 \\
10-20\end{array}$ & $\begin{array}{l}10,12 \\
10,16\end{array}$ & $\begin{array}{l}27,44 \\
27,37\end{array}$ & $\begin{array}{l}22,23 \\
21,40\end{array}$ & $\begin{array}{l}40,21 \\
41,06\end{array}$ & $\begin{array}{l}\text { Argila } \\
\text { Argila }\end{array}$ \\
\hline I tatiba & $\begin{array}{r}0-10 \\
10-20\end{array}$ & $\begin{array}{l}21,84 \\
19,71\end{array}$ & $\begin{array}{l}19,06 \\
17,16\end{array}$ & $\begin{array}{l}34,70 \\
40,60\end{array}$ & $\begin{array}{l}24,40 \\
22,53\end{array}$ & $\begin{array}{l}\text { Franco } \\
\text { Franco }\end{array}$ \\
\hline
\end{tabular}


25/02/2000. A instalação do filme plástico foi feita manualmente, após preparo e irrigação do solo, el iminando-se galhos eoutros materiais pontiagudos, para evitar danos capazes de prejudicar otratamento. O plástico foi esticado e fixado, enterrando-se as bordas em sulcos no solo, cobrindo toda a área da parcela tratada. Durantea solarização, a temperatura foi registrada, a $10 \mathrm{~cm}$ de profundidade, no canteiro central de cada parcela.

Pencycuron é indicado para o controle de $R$. solani e procimidone para o controle de Sclerotinia spp., tendo sido as aplicações dos fungicidas efetuadas aproximadamente 15 e 30 dias após o transplante das mudas de alface, ocorrido em 3/03/ 2000.

A resistência à penetração do solo foi avaliada em 6/04/2000, com auxílio de um penetrômetro CP20 ultrasonic cone penetrometer (Rimik). Foram amostrados quatro locais em cada parcela, até à profundidade de $20 \mathrm{~cm}$, sendo as leituras real izadas em intervalos de 2,5 cm. A umidade dos sol os foi obtida com medidor TDR (Time Domain Reflectometry da Trase Sistems), na profundidade de $0-20 \mathrm{~cm}$.

A análise química do solo foi feita, nas parcelas solarizadas e não solarizadas, em amostras compostas (mistura de 20 amostras simples), coletadas até $10 \mathrm{~cm}$ de profundidade, em 14/04/2000. $\mathrm{O} \mathrm{P}$ e os teores trocáveis de $\mathrm{K}^{+}, \mathrm{Ca}^{2+} \mathrm{e} \mathrm{M} \mathrm{g}^{2+}$ foram extraídos do sol o com resina de troca iônica; o B foi extraído com água quente; os micronutrientes metálicos (Cu, Fe, Mn e Zn), com solução de DTPATEA em pH 7,3, e o N-N ${ }_{4}^{+}$e N-N ${ }_{3}^{-}$, com solução de $\mathrm{KCl} 1 \mathrm{~mol} \mathrm{~L}-1$. O P eo B foram determinados por colorimetria; o $\mathrm{K}^{+}$, o $\mathrm{Ca}^{2+}$ e $\mathrm{O} \mathrm{Mg}^{2+}$, por espectr ofotometria de absorção atômica; o Cu, o Fe, o Mn e o Zn, por espectrofotometria de emissão atômica com plasma induzido e as formas inorgânicas deN por destilaçãoa vapor. Os métodos usados estão descritos em Raij et al. (2001).

A atividade microbiana do solo foi avaliada por meio da hidról ise de diacetato de fluoresceína (FDA), utilizando métodosemel hante ao descrito por Boehm \& Hoitink (1992), em 17/03/2000 e 28/06/2000. Amostras de $5 \mathrm{~g}$ de sol o foram col ocadas em frascos deErlenmeyer $(250 \mathrm{~mL}$ ), juntamentecom $20 \mathrm{~mL}$ de tampão fosfato de potássio $60 \mathrm{mmol} \mathrm{L}^{-1}(8,7 \mathrm{~g}$ de $\mathrm{K}_{2} \mathrm{HPO}_{4}$ e $1,3 \mathrm{~g}$ de $\mathrm{KH}_{2} \mathrm{PO}_{4}$ por $\mathrm{L}$ deágua destilada; pH 7,6). A reação de hidrólise de FDA (Sigma Chemical Co.) foi iniciada, adicionando-se $0,2 \mathrm{~mL}$ $(400 \mu \mathrm{g})$ de solução estoque de F DA ( $2 \mathrm{~g} \mathrm{~L}^{-1}$ acetona). As amostras foram incubadas por $20 \mathrm{~min}$ em agitador (200 rpm) a $25^{\circ} \mathrm{C}$. A reação foi interrompida pela adição de $20 \mathrm{~mL}$ deacetona por frasco. A seguir, procedeu-se à filtragem (Whatman no 1); os filtrados foram recol hidos em tubos decultura, posteriormente tampados com papel alumínio e acondicionados em recipiente que continha gelo, para evitar a evaporação da acetona. Em espectrofotômetro, determinou-sea absorbância (490 nm) dos filtrados.
A concentração de FDA hidrolisado $\left(\mu \mathrm{g} \mathrm{g}^{-1}\right.$ de FDA hidrol isado no sol o seco) foi determinada com auxílio de uma curva-padrão. A curva-padrão foi obtida adicionando-se FDA, nas quantidades de $0,100,200$, 300 e $400 \mu \mathrm{g}$, em $5 \mathrm{~mL}$ de tampão fosfato, contido em tubos de cultura. Os tubos foram mantidos por 60 min em água fervente, para hidrolisar o FDA. Após a hidrólise, o FDA foi então adicionado em Erlenmeyers que continham $5 \mathrm{~g}$ de sol o e $15 \mathrm{~mL}$ de tampão fosfato, em três repetições. A seguir, método semelhante ao descrito anteriormente foi utilizado com vista em obter a curva-padrão entre o FDA hidrolisado e a absorbância.

A supressividade a Fusarium oxysporum f. sp. phaseol $i$ foi avaliada pela colonização de amostras de solo pelo patógeno marcado com resistência a benomyl (10 $\left.\mathrm{mg} \mathrm{L}^{-1}\right)$, segundo o método de Toyota et al. (1996), com modificações. O solo, coletado em $6 / 04 / 2000$, atéà profundidade de $10 \mathrm{~cm}$, foi colocado em cilindros metáli cos desinfestados, com 4,5 cm de diâmetro e $4 \mathrm{~cm}$ dealtura, e cobertos com parafilme. Um disco de meio de cultura ( $1,5 \mathrm{~cm}$ de diâmetro) com micélio do fungo, retirado da borda de uma colônia, foi transferido para a superfície do solo. A incubação foi realizada a $25^{\circ} \mathrm{C}$, no escuro. Após 15 dias, o solo foi retirado dos cilindros, homogeneizado, sendo a avaliação da colonização pelo patógeno feita por meio de contagem das colônias desenvol vidas após plaqueamento em meio de cultura de Komada (1975), acrescido de benomyl (10 $\mathrm{mg} \mathrm{L}^{-1}$ ).

\section{J arinu}

O experimento foi instalado numa lavoura comercial no município de J arinu, produtora de morango. Os canteiros foram preparados eadubados antes da instal ação do experimento. O delineamento experimental foi em blocos ao acaso, com dois tratamentos (solarizado e não solarizado) e quatro repetições, constituídas por três canteiros de $1 \mathrm{~m}$ de largura, com $20 \mathrm{~cm}$ entre os canteiros, e $5 \mathrm{~m}$ de comprimento. Desse modo, as parcelas solarizadas foram cobertas com o plástico em área de $4 \times 5 \mathrm{~m}$. $\mathrm{O}$ plástico com $100 \mu$ m de espessura ecom aditivo antiUV foi colocado no sol o no dia 27/12/1999 e retirado no dia 28/02/2000, da forma descrita no experimento anterior.

A avaliação da resistência à penetração e da umidade do solo foi feita de forma semel hante ao ensaio de Mogi das Cruzes, em 18/04/2000 e 3/10/ 2000, tendo a área permanecido sem cultivo durante esse período. Na última avaliação foram retiradas amostras indeformadas de sol o, com cilindros de $5 \mathrm{~cm}$ de diâmetro e $6 \mathrm{~cm}$ de altura, nas profundidades de 10 e $20 \mathrm{~cm}$, em duas repetições por parcela. A macroporosidade, mi croporosidade, porosidadetotal e densidade foram avaliadas segundo EMBRAPA (1997). A avaliação de macro e micronutrientes foi feita como no ensaio anterior. 


\section{Piracicaba}

O experimento foi instaladona área experimental do Departamento de Horticultura da ESALQ/USP, em blocos casualizados, com quatro repetições. Foram avaliados três tipos de filmes plásticos para solarização, de $100 \mu$ m deespessura, produzidos pela empresa NORTENE Plásticos Ltda.: (a) filme plástico com aditivo anti-UV; (b) filme plástico com meia carga de aditivo anti-UV; (c) filme plástico transparente sem aditivo anti-UV. A testemunha foi constituída por parcela não solarizada.

As parcelas foram constituídas por três cantei ros com $5 \mathrm{~m}$ de comprimento. Os plásticos foram colocados no dia 18/01/2000 e retirados em 14/03/ 2000. Mudas de alface foram transplantadas em 14/ 04/2000. Durante a solarização, foram registradas as temperaturas do solo a $10 \mathrm{~cm}$ de profundidade no canteiro central das parcelas. As avaliações de macro e micronutrientes (em 14/04/2000), atividade microbiana (em 26/04/2000), resistência à penetração, macroporosidade, microporosidade, porosidadetotal e densidade (em 17/05/2000) foram efetuadas como nos experimentos anteriores.

\section{Itatiba}

O experimento foi instalado em área de cultivo comercial de morango, em blocos ao acaso, com quatro repetições. A área das parcelas foi a mesma do ensaio de J arinu. Os tratamentos foram: (a) solarização por 66 dias (plástico colocado em 12/12/ 2000 e retirado em 16/02/2001); (b) solarização por 119 dias (plástico colocado em 12/12/2000 e retirado em 10/04/2001); (c) solarização por 119 dias, tendo sido o plástico pintado com tinta preta e permanecido no campo durante o cultivo do morango; (d) testemunha não solarizada. O plantio do morango foi em 10/04/2001.

A resistência à penetração do solo e a umidade foram avaliadas em 28/03/2001, conforme descrito anteriormente.

\section{RESULTADOS E DISCUSSÃO}

No experimento de Mogi das Cruzes, as temperaturas médias do solo registradas na profundidade de $10 \mathrm{~cm}$ durante a solarização foram de 40,8 e $29,3^{\circ} \mathrm{C}$, para os sol os solarizados e não solarizados, respectivamente; as temperaturas máximas foram de 48 e $34^{\circ} \mathrm{C}$. Em Piracicaba, as temperaturas registradas não diferiram sob os diferentes filmes plásticos, sendo as médias de 30,6 e $25,4{ }^{\circ} \mathrm{C}$, para os sol os solarizados e não solarizados, respectivamente, e as máximas de 43,9 e $33,9^{\circ} \mathrm{C}$. As temperaturas obtidas indicam que os experimentos foram realizados em períodos adequados para solarização (Ghini et al., 1994).
A solarização reduziu significativamente a resistência à penetração dos solos nos ensaios de J arinu, Piracicaba el tatiba (Figura 1). EmJ arinu, onde o solo apresentou maior resistência inicial à penetração, a diferença entre os tratamentos foi muito acentuada. Oito meses após a retirada do plástico, verificou-se que o efeito da sol arização sobre essa característica física do sol o não foi al terado. No ensaio de Piracicaba, os plásticos com diferentes cargas de aditivo anti-UV apresentaram resultados semel hantes, assim como no ensaio de I tatiba, em que os vários períodos de tratamento também não diferiram quanto aos efeitos na resistência à penetração dos sol os (Figura 1).

Embora a resistência à penetração esteja relacionada de forma inversa com a umidade do solo, em J arinu, Piracicaba e I tatiba, a variação na umidade do solo por si só não foi suficiente para explicar tal diferenciação observada. Os solos não solarizados apresentaram maior resistência à penetração e mai or umidade(Figura 1). Observando os val ores de densidade, verifica-se que, Piracicaba e J arinu, locais onde foram feitas as avaliações, apresentaram valores menores para os solos solarizados (Quadro 2), o que pode explicar a menor resistência à penetração. De modo geral, a variação na macro, micro e porosidade total não foi significativa entre os tratamentos solarizados e não solarizados (Quadro 2). Por outrolado, Patel \& Patel (1997) verificaram, em um solo de textura francoarenosa, aumento na porosidade total e na capacidade de retenção de água, após a sol arização, sem, entretanto, explicar os fatores envolvidos nessas alterações.

No ensaio de Mogi das Cruzes, em sol o turfoso, a solarização resultou em aumento da resistência à penetração do solo na camada de 2,5 a $5 \mathrm{~cm}$ de profundidade $(97,45$ e $42,44 \mathrm{kPa}$, nos solos solarizados e não solarizados, respectivamente) (Figura 1). Nesse caso, a resistência à penetração foi inversamente relacionada com a umidade dos sol os, como era esperado. As maiores temperaturas durante a solarização concentram-se na superfície do solo, provocam maior ressecamento da matéria orgânica e acarretam, conseqüentemente, aumento da resistência à penetração.

A atividade microbiana, avaliada pela hidrólise de diacetato de fluoresceína, foi reduzida pela solarização, nos ensaios de Mogi das Cruzes e Piracicaba (Quadro 3). Resultado semel hante foi obtido por Schoenmaker (2001), em ensaio realizado com solarização para o controle de Pythium em crisântemo e pepino. O aquecimento do solo durante a solarização promoveu a morte de uma parcela de microrganismos, o que podeter resultado na redução da atividade microbiana. Os fungicidas não apresentaram efeito, graças, possivelmente, à degradação dos produtos ou ao modo de ação específico (Lyr, 1995), podendo ter atuado sobre uma 
(a) Mogi das Cruzes

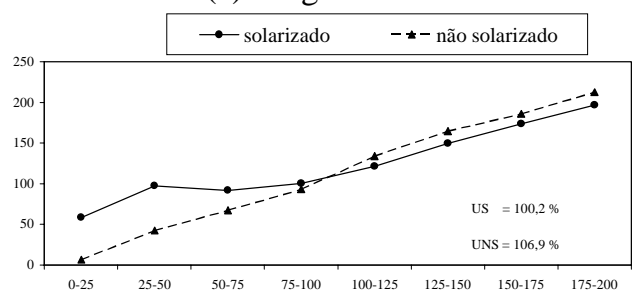

(b) Jarinu 18/04/2000

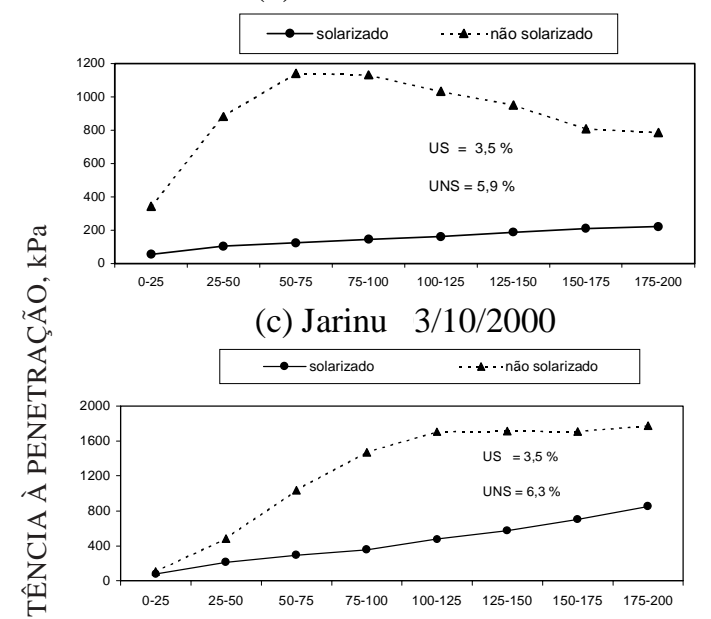

(d) Piracicaba

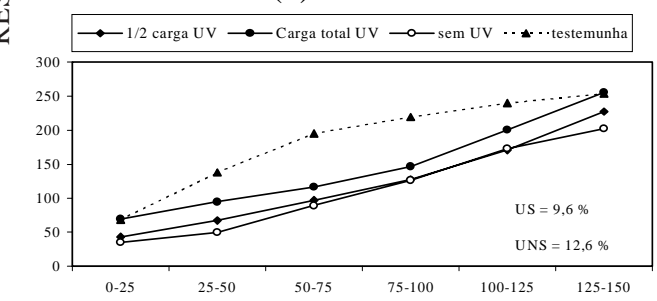

(e) Itatiba

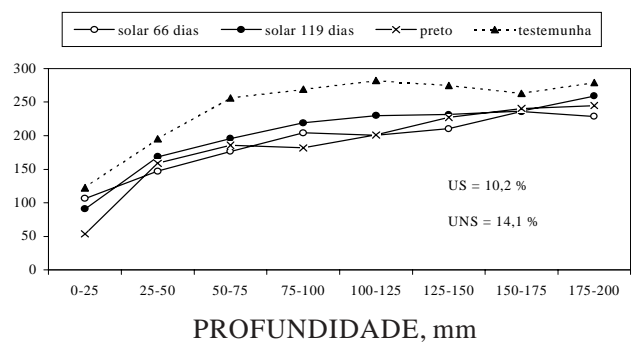

Figura 1. Resistência à penetração dos solos submetidos à solarização, nos ensaios de (a) Mogi das Cruzes, (b) J arinu em 18/04/2000, (c) J arinu em 3/10/2000, (d) Piracicaba (1/2 carga UV = solarização com plástico com metade da carga de aditivo anti-UV; carga total UV = solarização com plástico com carga total de aditivo anti-UV e sem UV = solarização com plástico transparente sem aditivo) e (e) I tati ba (solar 66 dias = solarização por 66 dias; solar 119 dias = solarização por 119 dias; preto = solarização seguida de pintura do plástico com tinta preta; testemunha = não solarizado). US = umidade do solo solarizado e UNS = umidade do solo não solarizado. parte pouco significativa da microbiota do solo, sem causar alterações na atividade microbiana total. A hidrólise de FDA é considerada uma avaliação da atividade microbiana geral, visto que a reação pode ser realizada pela maioria das bactérias, fungos e outros organismos (Grunwal d et al ., 2000). A alteração na microbiota do solo pode ter contribuído para a redução na resistência à penetração, considerando que os mi crorganismos presentes no sol o interagem com as partículas de argila tendo influência na estabilização dos agregados (Tsai et al., 1992).

A supressividade a $F$. oxysporum f. sp. phaseol $i$ foi aumentada com a solarização, pois o tratamento reduziu a recuperação do patógeno (Quadro 4). Segundo Alabouvette et al. (1996), a supressão a Fusarium pode ser resultante do aumento da atividade microbiana total ou de natureza específica. A supressão observada nos solos solarizados pode ter sido de natureza específica, já que não foi acompanhada do aumento da atividade mi crobiana total. Ou seja, as alterações promovidas pela solarização na composição da microbiota do solo podem ter resultado na seleção de antagonistas específicos. Os fungicidas não afetaram a recuperação do patógeno, pois, além de não serem recomendados para o controle de Fusariumspp., não promoveram al terações que resultassem em indução de supressividade (Quadros 3 e 4).

Nos sol os solarizados, houve aumento significativo dos teores de $\mathrm{NH}_{4}{ }^{+}$em todos os ensaios e $\mathrm{Mn}$, em três ensaios. $\mathrm{Em}$ alguns experimentos, foram observados aumentos dos teores de $\mathrm{NO}_{3}{ }^{-}, \mathrm{Mg}^{2+}, \mathrm{K}^{+} \mathrm{e}$ da saturação por bases, além da redução dos teores de H + Al, Cu, Fe e Zn (Quadro 5). O aumento acentuado nos teores de $\mathrm{NH}_{4}{ }^{+}$deve estar relacionado com o incremento na mineralização do N orgânico do sol o pelo aumento da temperatura, o que favorece também a liberação de $\mathrm{Mn}$ e $\mathrm{K}$. Outros autores também observaram aumentos nos teores de $\mathrm{NH}_{4}+$, $\mathrm{NO}_{3}{ }^{-}, \mathrm{K}+, \mathrm{Ca}^{2+} \mathrm{eMg}^{2+}$ nos solos solarizados (Stapleton et al., 1985; Katan \& DeVay, 1991; Patel \& Patel, 1997; Grunzweig et al., 1999). O incremento na disponibilidade de formas inorgânicas de $\mathrm{N}$ geralmente estimula o crescimento das plantas, pois esse é o elemento mineral absorvido em maiores quantidades pela maioria dos vegetais. Segundo Grunzweig et al. (1999), o efeito da solarização nos nutrientes tem diversas implicações, especialmente em sol os pobres. Elementos comoK emicronutrientes, como $\mathrm{Mn}$, podem promover a redução da incidência de doenças de plantas, por meio da indução de supressividade do solo a patógenos, indução da resistência de plantas ou outros mecanismos (Engelhard, 1989).

O teor de matéria orgânica não diferiu nos quatro ensaios (Quadro 5). E mbora tenha havido liberação de N inorgânico com a solarização, as quantidades liberadas são muito pequenas em relação ao estoque deN da matéria orgânica. Assim, no curto intervalo 
Quadro 2. Macroporosidade, microporosidade, porosidade total e densidade de solos submetidos à solarização, nas profundidades de 10 e 20 cm, nos ensaios de Piracicaba e J arinu (SP)

\begin{tabular}{|c|c|c|c|c|}
\hline Tratamento & Macroporosidade & Microporosidade & Porosidade total & Densidade \\
\hline & & $-\%$ & 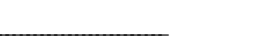 & $\mathrm{kg} \mathrm{dm}^{-3}$ \\
\hline \multicolumn{5}{|l|}{ Piracicaba $(17 / 05 / 2000)$} \\
\hline $10 \mathrm{~cm}$ & $30 \mathrm{a}$ & $26 \mathrm{~b}$ & 56 a & $1,16 \mathrm{~b}$ \\
\hline $20 \mathrm{~cm}$ & $26 \mathrm{a}$ & $31 \mathrm{a}$ & 58 a & $1,29 a$ \\
\hline \multicolumn{5}{|l|}{ Solarização } \\
\hline Testemunha & 28 a & 31 a & 59 a & $1,32 \mathrm{a}$ \\
\hline Solarizado - carga total UV & $29 a$ & $27 \mathrm{~b}$ & $57 a$ & $1,19 \mathrm{ab}$ \\
\hline Solarizado - meia carga UV & $28 \mathrm{a}$ & $28 \mathrm{ab}$ & 56 a & $1,18 \mathrm{~b}$ \\
\hline Solarizado - sem UV & & $30 a b$ & 57 a & $1,22 a b$ \\
\hline Testemunha - $10 \mathrm{~cm}$ & 29 & 30 & 59 & 1,28 \\
\hline Solarizado - carga total UV - $10 \mathrm{~cm}$ & 31 & 24 & 56 & 1,13 \\
\hline Solarizado - meia carga UV - $10 \mathrm{~cm}$ & 30 & 24 & 54 & 1,07 \\
\hline Solarizado - sem UV - $10 \mathrm{~cm}$ & 29 & 27 & 57 & 1,17 \\
\hline Testemunha - $20 \mathrm{~cm}$ & 26 & 33 & 59 & 1,35 \\
\hline Solarizado - carga total UV - $20 \mathrm{~cm}$ & 28 & 29 & 58 & 1,26 \\
\hline Solarizado - meia carga UV - $20 \mathrm{~cm}$ & 26 & 32 & 58 & 1,29 \\
\hline Solarizado - sem UV - $20 \mathrm{~cm}$ & 25 & 32 & 57 & 1,27 \\
\hline \multicolumn{5}{|l|}{$J$ arinu (3/10/2000) } \\
\hline Solarizado - $10 \mathrm{~cm}$ & 31,38 a & 28,95 a & 60,33 a & $1,09 \mathrm{~b}$ \\
\hline Não sol arizado $-10 \mathrm{~cm}$ & 32,75 a & $29,34 \mathrm{a}$ & 62,10 a & $1,09 \mathrm{~b}$ \\
\hline Solarizado - $20 \mathrm{~cm}$ & 31,99 a & 29,04 a & 61,04 a & $1,05 \mathrm{~b}$ \\
\hline Não solarizado - $20 \mathrm{~cm}$ & 32,80 a & $30,69 \mathrm{a}$ & 63,49 a & 1,16 a \\
\hline
\end{tabular}

Médias seguidas da mesma letra, nas colunas, não diferem entre si pelo teste de Tukey a 1 \%.

Quadro 3. Atividade microbiana do solo, avaliada pela hidrólise de diacetato de fluoresceína (FDA) dos solos submetidos à solarização, nos ensaios de Mogi das Cruzes e Piracicaba (SP)

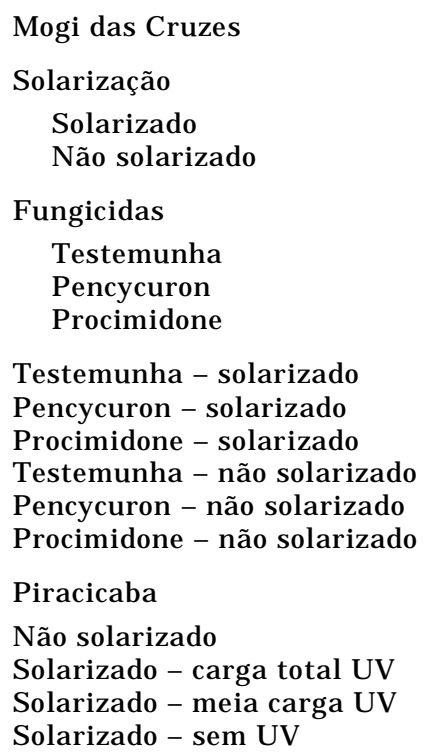

- $\mu \mathrm{g} \mathrm{g}^{-1}$ de FDA hidrolisado no solo seco -

\section{$17 / 03 / 2000$}

$28 / 06 / 2000$

$$
14,48 \mathrm{~b}
$$

$18,06 \mathrm{a}$
$18,06 \mathrm{a}$
$19,09 \mathrm{a}$
13,49
14,75
15,20
22,63
21,38
22,97
$26 / 04 / 2000$
$8,65 \mathrm{a}$
$0,76 \mathrm{c}$
$1,14 \mathrm{bc}$
$1,89 \mathrm{~b}$


de tempo do experimento, não poderiam ocorrer variações no teor de matéria orgânica do solo. Resultados semel hantes foram obtidos por Stapleton et al. (1985), porém os autores advertem que as discrepâncias observadas entre os resultados dos efeitos da solarização nos nutrientes do solo se devem, em parte, aos diferentes tipos de solo, profundidade de amostragem e métodos empregados para análise.

Os efeitos observados da solarização, como a redução da resistência à penetração, indução de supressividade, liberação de nutrientes, especialmente $\mathrm{N}$, podem contribuir para maior crescimento de plantas, controle de patógenos veiculados pelo solo e, conseqüentemente, maior produtividade. Assim, a solarização apresenta grande potencial no manejo de culturas e, por esse motivo, outros estudos devem ser realizados para melhor caracterização e explicação das causas da variação.
Quadro 4. E feito da solarização na colonização de solos de Mogi das Cruzes (SP) por Fusarium oxysporum f. sp. phaseoli

\begin{tabular}{lc}
\hline \multicolumn{1}{c}{ Tratamento } & log ufc/g solo seco \\
\hline Solarização & \\
$\quad$ Solarizado & $2,80 \mathrm{~b}$ \\
Não solarizado & $3,32 \mathrm{a}$ \\
Fungicidas & \\
Testemunha & $3,09 \mathrm{a}$ \\
Pencycuron & $2,90 \mathrm{a}$ \\
Procimidone & $3,19 \mathrm{a}$ \\
Testemunha - solar & 2,95 \\
Pencycuron - solar & 2,51 \\
Procimidone - solar & 2,93 \\
Testemunha - não solar & 3,24 \\
Pencycuron - não solar & 3,29 \\
Procimidone - não solar & 3,45
\end{tabular}

Médias seguidas da mesma letra não diferem entre si pelo teste de Tukey a $1 \%$.

Quadro 5. Composição química dos solos submetidos à solarização nos ensaios de Mogi das Cruzes, J arinu, Piracicaba e Itatiba (SP)

\begin{tabular}{|c|c|c|c|c|c|c|c|c|c|c|c|c|c|c|c|c|}
\hline Local & Tratamento & MO & $\underset{\left(\mathrm{CaCl}_{2}\right)}{\mathbf{p H}}$ & $\mathrm{NH}_{4}^{+}$ & $\mathrm{NO}_{3}^{-}$ & $\mathbf{P}$ & $\mathbf{K}^{+}$ & $\mathrm{Ca}^{2+}$ & $\mathrm{Mg}^{2+}$ & $\mathbf{H}+\mathbf{A} \mathbf{I}$ & $\mathbf{v}$ & B & $\mathbf{C u}$ & $\mathbf{F e}$ & Mn & Zn \\
\hline & & \multicolumn{2}{|l|}{$\mathrm{gdm}^{3}$} & \multicolumn{3}{|c|}{$\mathrm{mg} \mathrm{dm}^{-3}$} & 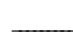 & \multicolumn{3}{|c|}{$-\mathrm{mmol}_{\mathrm{c}} \mathrm{dm}^{-3}$} & $\%$ & & & \multicolumn{2}{|c|}{$-m g d m^{-3}$} & \\
\hline Mogi das & Solarizado & 218 & $5,9^{(1)}$ & $119,6^{(1)}$ & 46,0 & 1156 & $7,9^{(1)}$ & 375 & $59^{(1)}$ & 39 & $91^{(1)}$ & 3,70 & 20,5 & 87 & $107,0^{(1)}$ & 46,4 \\
\hline Cruzes & Não sol arizado & 202 & 5,7 & 5,4 & 41,1 & 1417 & 6,5 & 383 & 54 & $53^{(1)}$ & 89 & 3,00 & $25,4^{(1)}$ & $119^{(1)}$ & 32,9 & $67,9^{(1)}$ \\
\hline \multirow[t]{2}{*}{ J arinu } & Solarizado & 22 & 5,6 & $62,6^{(1)}$ & $45,0^{(1)}$ & 194 & 6,1 & 34 & 12 & 23 & 68 & $1,14^{(1)}$ & 1,4 & 24 & $11,3^{(1)}$ & 4,5 \\
\hline & Não sol arizado & 21 & 5,6 & 1,0 & 18,1 & 158 & 5,2 & 29 & 10 & 23 & 65 & 0,61 & 1,6 & 27 & 7,6 & 4,8 \\
\hline \multirow[t]{2}{*}{ Piracicaba } & Solariz & 29 & $5,9^{(1)}$ & $100,3^{(1)}$ & 31,1 & 154 & 9,2 & 59 & $22^{(1)}$ & 25 & $78^{(1)}$ & 0,29 & 15,2 & 54 & $211,4^{(1)}$ & 17,2 \\
\hline & Não solarizado & 26 & 5,5 & 34,2 & 39,5 & 186 & 7,2 & 54 & 19 & $33(1)$ & 70 & 0,29 & $18,3^{(1)}$ & $86^{(1)}$ & 108,6 & 16,9 \\
\hline \multirow[t]{2}{*}{ Itatiba } & Solarizado & 23 & 5,9 & $24,4^{(1)}$ & $51,3^{(1)}$ & 104 & 5,5 & 47 & 10 & 21 & 74 & 0,45 & 3,2 & 42 & 23,2 & 4,5 \\
\hline & Não sol arizado & 24 & 6,0 & 2,6 & 3,8 & 152 & 5,3 & 45 & 10 & 22 & 72 & 0,37 & 2,8 & 40 & 18,9 & 4,2 \\
\hline
\end{tabular}

(1) Médias diferem significativamente, entre solos solarizados ou não, dentro de cada local, pelo teste de Tukey a 5 \%.

\section{CONCLUSÕES}

1. A solarização reduziu significativamente a resistência à penetração dos solos não turfosos.

2. A atividade microbiana do solo foi reduzida pela solarização.

3. A supressividadea Fusarium oxysporumf. sp. phaseoli foi induzida pela solarização.

4. A solarização provocou um aumento expressivo nos teores de $\mathrm{NH}_{4}{ }^{+}$e de $\mathrm{Mn}$.

\section{AGRADE CIMENTOS}

À F undação de Amparo à Pesquisa do estado de São Paulo (FAPESP), pelo auxílio financeiro que possibilitou a realização dos experimentos. Ao produtor Sr. Mário Okuyama, pela condução do experimento em Mogi das Cruzes. AoEngoAgroj osé Abrahão Haddad Galvão, pel o auxílio nas avaliações.

\section{LITE RATURA CITADA}

ALABOUVETTE, C.; LEMANCEAU, P. \& STEINBERG, C. Biological control of Fusarium wilts: opportunities for developing a commercial product. In: HALL, R., ed. Principles and practice of managing soilborne plant pathogens. St. Paul, APS Press, 1996. p.192-212.

BETTIOL, W.; GHINI, R.; GALVÃO, J.A.H. \& ZOCCHI, S.S. Solarização do solo para o controle de Pythium e plantas daninhas em crisântemo. Sci. Agric., 51:459-462, 1994. 
BOEHM, M.J. \& HOITINK, H.A.J. Sustenance of microbial activity in potting mixes and its impact on severity of Pythium root rot of Poinsettia. Phytopathology, 82:259-264, 1992.

EMPRESA BRASILEIRA DE PESQUISA AGROPECUÁRIA EMBRAPA. Centro Nacional de Pesquisa de Solos. Manual de métodos de análise de solo. 2.ed. Rio de J aneiro, 1997. $212 p$.

ENGELHARD, A.W. Management of diseases with macro- and microelements. St. Paul, APS Press, 1989. 217p.

GHINI, R. \& BETTIOL, W. Controle físico. In: BERGAMIN FILHO, A.; KIMATI, H. \& AMORIM, A., eds. Manual de fitopatologia. 3.ed. São Paulo, Agronômica Ceres, 1995. p.786-803.

GHINI, R.; BETTIOL, W. \& SOUZA, N.L. Solarização do solo para o controle de Verticillium dahliae em berinjela. Fitopatol. Bras., 17:384-388,1992.

GHINI, R.; PARAIBA, L.C. \& LIMA, M.W.P. Determinação de período para solarização do solo na região de Campinas (SP). Summa Phytopathol., 20:131-133, 1994.

GRUENZWEIG, J.M.; RABINOWITCH, H.D. \& KATAN, J. Physiological and developmental aspects of increased plant growth in solarised soils. Ann. Appl. Biol., 122:579-591, 1993.

GRUNWALD, N.J .; HU, S. \& van BRUGGEN, A.H.C. Short-term cover crop decomposition in organic and conventional soils: caracterization of soil C, N, microbial and plant pathogen dynamics. Eur. J. Plant Pathol., 106:37-50, 2000.

KATAN, J. Solar heating (solarization) of soil for control of soilborne pests. Ann. Rev. Phytopathol., 19:211-236, 1981.

KATAN, J . Soil solarization: integrated control aspects. I n: HALL, R., ed. Principles and practice of managing soilborne plant pathogens. St. Paul, APS, 1996. p.250-278.

KATAN, J. \& DeVAY, J.E. Soil solarization. Boca Raton, CRC Press, 1991. 267p.

KATAN, J.; GREENBERGER, A.; ALON, H. \& GRINSTEIN, A. Solar heating by polyethylene mulching for the control of diseases caused by soil-borne pathogens. Phytopathology, 66:683-688, 1976.
KOMADA, H. Development of a selective medium for quantitative isolation of Fusarium oxysporumfrom natural soil. Rev. Plant Protec. Res., 8:114-125, 1975.

LIFSHITZ, R.; TABACHNIK, M.; KATAN, J. \& CHET, I. The effect of sublethal heating on sclerotia of Sclerotium rolfsii. Can. J. Microbiol., 29:1607-1610, 1983.

LYR, H. Modern selective fungicides: properties, applications, mechanisms of action. New York, Gustav Fisher, 1995. 595p.

PATEL, B.K. \& PATEL, H.R. Effect of nematicides, soil solarization, rabbing and green manuring on some physical and chemical properties of soil in nematode infested bidi tobacco nursery. Tob. Res., 23:19-26, 1997.

PREVEDELLO, C.L. Física do solo com problemas resolvidos. Curitiba, SAEAFS, 1996. 446p.

RAIJ , B. van;ANDRADE, J .C.; CANTARELLA, H. \& QUAGGIO, J.A. Análise química para avaliação da fertilidade de solos tropicais. Campinas, Instituto Agronômico, 2001. 284p.

SCHOENMAKER, I.A.S. Solarização do solo associada à incorporação de matérias orgânicas para o controle de Pythium spp. Piracicaba, Escola Superior de Agricultura "Luiz de Queiroz", 2001. 50p.

SINIGAGLIA, C.; PATRICIO, F.R.A.; GHINI, R.; MALAVOLTA, V.M.A.; TESSARIOLI, J. \& FREITAS, S.S. Controle de Sclerotinia minor, Rhizoctonia solani e plantas daninhas em alface pela solarização do solo e sua integração com controle químico. Summa Phytopathol., 27:229-235, 2001.

SOUZA, N.L. Solarização do solo. Summa Phytopathol., 20:315, 1994.

STAPLETON, J J .; QUICK, J. \& DeVAY, J.E. Soil solarization: effects on soil properties, crop fertilization and plant growth. Soil Biol. Biochem., 17:369-373, 1985.

TOYOTA, K.; RITZ, K.\& YOUNG, I.M. Microbiological factors affecting the colonization of soil aggregates by Fusarium oxysporumf. sp. raphani. Soil Biol. Biochem., 28:1513-1521, 1996.

TSAI, S.M.; BARAIBAR, A.V.L. \& ROMANI, V.L.M. Efeito de fatores do solo. In: CARDOSO, E.J.B.N.; TSAI, S.M. \& NEVES, M.C.P., eds. Microbiologia do solo. Campinas, 1992. p.59-72. 